\begin{tabular}{lll}
\hline & Physics Procedia & $\because$ \\
CrossMark & Volume 75, 2015, Pages 332-339 & \\
20th International Conference on Magnetism & Physics \\
ELSEVIER & Procedia \\
\hline
\end{tabular}

\title{
Competition of Spin and Charge Orders in a Model Cuprate
}

\author{
Yury D. Panov ${ }^{1}$, Alexander S. Moskvin ${ }^{1}$, Alexander A. Chikov ${ }^{1}$, and Ilya L. \\ Avvakumov $^{1}$ \\ Ural Federal University, Ekaterinburg, Russia \\ yuri.panov@urfu.ru
}

\begin{abstract}
We present results of the mean-field analysis of the competition between charge and spin orders in a model cuprate. Phase diagrams and temperature dependencies of the charge and magnetic order parameters are calculated for different doping given different values of the on-site correlation parameter, inter-site density-density coupling, and Heisenberg spin exchange integral.
\end{abstract}

Keywords: Cuprates, Spin and Charge Ordering, Mean Field

\section{Introduction}

The mechanism underlying the high-temperature superconductivity of copper oxides has remained unelucidated and is still one of the greatest mysteries in the field of condensed matter physics. The cuprate high- $T_{c}$ superconductors start out life as antiferromagnetic insulators in contrast with BCS superconductors being conventional metals. Unconventional behavior of these materials under charge doping, in particular, a remarkable interplay of charge, lattice, orbital, and spin degrees of freedom (see review article Ref.[1] and references therein), strongly differs from that of ordinary metals and merely resembles that of a doped Mott insulator. The fascinating issue of competing orders or intertwining effect between bulk superconductivity, charge density waves and static magnetic order in the cuprate superconductors has attracted a lot of attention for many years, however, the mechanisms are still unclear at present. In view of this, working models which can reproduce several aspects of this interplay can certainly contribute to a better understanding of the mechanisms at play. Here we address the competition between charge and spin orders in a model cuprate and present both analytical and numerical results of the mean-field approximation (MFA).

The rest of the paper is organized as follows. In Sec.2, we shortly consider an $\mathrm{S}=1$ pseudospin formalism to describe the charge degree of freedom in cuprates and introduce a simplified Hamiltonian for a model cuprate. Section 3 is devoted to the MFA analysis of the Hamiltonian 
on a square lattice. In Sec.4, we present the MFA phase diagrams, showing temperature vs. onsite correlation parameter and temperature vs. doping, as well as the temperature dependencies of the charge and spin order parameters. In Sec.5, we shortly summarize our results.

\section{$2 \quad S=1$ pseudospin formalism and model cuprate}

We start with a minimal model [2] of the copper oxides such as $\mathrm{La}_{2-x} \mathrm{Sr}_{x} \mathrm{CuO}_{4}$ with the on-site Hilbert space reduced to only three effective valence states (nominally $\mathrm{Cu}^{1+; 2+; 3+}$ ) of copper ions in the $\mathrm{CuO}_{2}$ planes. Central point of the model implies the occurrence of unconventional on-site quantum superpositions of the three valence states

$$
|\mathbf{c}\rangle=c_{-1}\left|C u^{1+}\right\rangle+c_{0}\left|C u^{2+}\right\rangle+c_{+1}\left|C u^{3+}\right\rangle,
$$

characterized by different hole occupation: $n_{h}=0,1,2$ for $\mathrm{Cu}^{1+; 2+; 3+}$ centers, respectively, and different conventional spin: $s=\frac{1}{2}$ for $\mathrm{Cu}^{2+}$ center and $s=0$ for $\mathrm{Cu}^{1+; 3+}$ centers.

The three different valence charge states of the $\mathrm{Cu}^{1+; 2+; 3+}$ centers are associated with the three components of the $S=1$ pseudo-spin triplet with $M_{S}=-1,0,+1$, respectively, so the local hole density $n_{h}$ and the doped hole concentration $n$ are related with the pseudomagnetization and read as follows:

$$
n_{h} \rightarrow S_{i z}+1, \quad n=\frac{1}{N} \sum_{i=1}^{N}\left\langle S_{i z}\right\rangle
$$

Conventional spin density for mixed valence superpositions can vary inbetween 0 and 1 in accordance with the weight of the $\mathrm{Cu}^{2+}$ center in the on-site superposition. Projection operator $\hat{P}_{0}=\hat{\rho}^{s}=\left(1-\hat{S}_{z}^{2}\right)$ picks out the $s=\frac{1}{2} \mathrm{Cu}^{2+}$ center in the on-site mixed valence superpositions (1) and can be addressed to be the on-site spin density operator.

Effective $S=1$ pseudo-spin Hamiltonian which does commute with the z-component of the total pseudo-spin $\sum_{i} S_{i z}$ can be written to be a sum of the three terms [3]:

$$
\hat{H}=\hat{H}_{p o t}+\hat{H}_{k i n}^{(1)}+\hat{H}_{k i n}^{(2)},
$$

where $\hat{H}_{p o t}$ includes the on-site and inter-site potential energy terms, while $\hat{H}_{k i n}^{(1)}$ and $\hat{H}_{k i n}^{(2)}$ present the one- and two-particle inter-site hopping terms. A comprehensive analysis of the full Hamiltonian for cuprates remains to be a longstanding challenge for the condensed matter community. However, to uncover some specific features of the spin-charge competition, hereafter, in the paper we shall consider only simplified spin-pseudo-spin Hamiltonian which takes into account the on-site and inter-site correlations, that is potential energy terms $\hat{H}_{\text {pot }}$, and conventional Heisenberg spin exchange coupling:

$$
\hat{H}=\Delta \sum_{i=1}^{N} S_{i z}^{2}-\mu \sum_{i=1}^{N} S_{i z}+\sum_{\langle i j\rangle} V_{i j} S_{i z} S_{j z}+\sum_{\langle i j\rangle} \hat{I}_{i j}\left(\vec{s}_{i}, \vec{s}_{j}\right)
$$

where the sums run over sites of a two-dimensional square lattice, $\langle i j\rangle$ means the nearest neighbors, $S_{i z}$ and $\vec{s}_{i}$ are the on-site pseudo-spin and conventional spin operators, respectively.

The first on-site term ("single-ion anisotropy") describes the effects of a bare "pseudospin splitting" and relates with the on-site density-density interactions: $\Delta=U / 2, U$ being the correlation parameter, the second term, or a pseudospin Zeeman coupling, with chemical 
potential $\mu$ is needed to account for the charge density constraint, the third (Ising) term with $V>0$ describes the effects of the inter-site density-density interactions. The last term is the antiferromagnetic $\mathrm{Cu}^{2+}-\mathrm{Cu}^{2+}$ Heisenberg spin exchange coupling, where instead of the conventional bare exchange integral $I_{i j}$ we arrive at an effective pseudo-spin operator

$$
\hat{I}_{i j}=\hat{P}_{0 i} I_{i j} \hat{P}_{0 j}=\hat{\rho}_{i}^{s} I_{i j} \hat{\rho}_{j}^{s}=\left(1-\hat{S}_{i z}^{2}\right) I_{i j}\left(1-\hat{S}_{j z}^{2}\right),
$$

that takes into account the on-site occupation dependence. It is worth noting that the existence of localized spins on the $\mathrm{Cu}^{2+}$ ions persisting up to slightly overdoping was ascertained by Johnston [4] not long after the discovery of the cuprate superconductors. Unconventional temperature and doping behavior of the localized spin contribution to magnetic susceptibility $\chi^{2 D}(T)$ was attributed to a rapid drop both of the intralayer $\mathrm{Cu}-\mathrm{Cu}$ exchange integrals and effective $\mathrm{Cu}$ spin moments with rising doping. However, the pseudospin operator form of the effective exchange integral (5) points to a just another and more justified cause of the puzzle around $\chi^{2 D}(T)$, that is a rapid drop of the on-site $\mathrm{Cu}^{2+}$ fraction and, accordingly, on-site spin density $\hat{\rho}^{s}$.

Obviously, both the on-site repulsion $\Delta>0$ and conventional spin exchange provide an energy gain to the parent antiferromagnetic insulating (AFMI) phase with $\left\langle\hat{S}_{i z}^{2}\right\rangle=0$, while both the on-site attraction $\Delta<0$ and inter-site coupling $V>0$ do promote the charge order thus driving the competition of the spin and charge orders.

\section{Mean field approximation}

Hereafter, we shall be concerned with a mean-field analysis of the model Hamiltonian (4) for a $2 \mathrm{D}$ square lattice with the nearest-neighbor inter-site coupling, $V_{n n}=V, I_{n n}=I$ confining ourselves to the simplest two-sublattice $(A$ and $B)$ approximation.

The Bogolubov inequality is used to estimate the grand potential $\Omega$ :

$$
\Omega(H) \leq \Psi=\Omega\left(H_{0}\right)+\left\langle H-H_{0}\right\rangle .
$$

Within the two-sublattice model we take

$$
\frac{H_{0}}{T}=\delta \sum_{i} S_{i z}^{2}-\sum_{\alpha, i_{\alpha}} \beta_{\alpha} S_{i_{\alpha} z}-\sum_{\alpha, i_{\alpha}} \gamma_{\alpha} P_{0 i_{\alpha}} s_{i_{\alpha} z},
$$

where $\delta=\Delta / T, \beta_{\alpha}$ and $\gamma_{\alpha}$ are variational parameters (molecular fields), $\alpha=A, B$. The mean field estimate of the grand potential takes the form

$$
\frac{2 \Psi}{T N}=\sum_{\alpha}\left[\left(\beta_{\alpha}-\xi\right) S_{\alpha}+\gamma_{\alpha} \sigma_{\alpha}-\log \left(e^{-\delta} \cosh \beta_{\alpha}+\cosh \gamma_{\alpha}\right)\right]+4 \nu S_{A} S_{B}+j \sigma_{A} \sigma_{B}-\log 4
$$

where $\xi=\mu / T, \nu=V / T, j=I / T$ ( $V$ and $I$ are density-density coupling parameter and bare exchange integral for nearest neighbors), and

$$
S_{\alpha}=\frac{\sinh \beta_{\alpha}}{\cosh \beta_{\alpha}+e^{\delta} \cosh \gamma_{\alpha}}, \quad \sigma_{\alpha}=\frac{\sinh \gamma_{\alpha}}{e^{-\delta} \cosh \beta_{\alpha}+\cosh \gamma_{\alpha}}
$$

are the mean values of the $z$-component of pseudospin and conventional spin on $\alpha$-sublattice, that is order parameters: $\left|S_{A, B}\right| \leq 1,\left|\sigma_{A, B}\right| \leq 1$. Minimization of $\Psi$ with respect to $\beta_{\alpha}$ and $\gamma_{\alpha}$ 
gives the mean field equations:

$$
\left\{\begin{aligned}
\beta_{A}-\xi & =-\frac{4 \nu \sinh \beta_{B}}{\cosh \beta_{B}+e^{\delta} \cosh \gamma_{B}}, \\
\beta_{B}-\xi & =-\frac{4 \nu \sinh \beta_{A}}{\cosh \beta_{A}+e^{\delta} \cosh \gamma_{A}}, \\
\gamma_{A} & =-\frac{j \sinh \gamma_{B}}{e^{-\delta} \cosh \beta_{B}+\cosh \gamma_{B}}, \\
\gamma_{B} & =-\frac{j \sinh \gamma_{A}}{e^{-\delta} \cosh \beta_{A}+\cosh \gamma_{A}}, \\
2 n & =\frac{\sinh \beta_{A}}{\cosh \beta_{A}+e^{\delta} \cosh \gamma_{A}}+\frac{\sinh \beta_{B}}{\cosh \beta_{B}+e^{\delta} \cosh \gamma_{B}} .
\end{aligned}\right.
$$

In terms of order parameters $S_{\alpha}$ and $\sigma_{\alpha}$, we obtain

$$
\left\{\begin{aligned}
S_{A} & =\frac{\sinh \left(\xi-4 \nu S_{B}\right)}{\cosh \left(\xi-4 \nu S_{B}\right)+e^{\delta} \cosh j \sigma_{B}}, \\
S_{B} & =\frac{\sinh \left(\xi-4 \nu S_{A}\right)}{\cosh \left(\xi-4 \nu S_{A}\right)+e^{\delta} \cosh j \sigma_{A}}, \\
\sigma_{A} & =-\frac{\sinh j \sigma_{B}}{e^{-\delta} \cosh \left(\xi-4 \nu S_{B}\right)+\cosh j \sigma_{B}}, \\
\sigma_{B} & =-\frac{\sinh j \sigma_{A}}{e^{-\delta} \cosh \left(\xi-4 \nu S_{A}\right)+\cosh j \sigma_{A}}, \\
2 n & =S_{A}+S_{B} .
\end{aligned}\right.
$$

The last equation accounts for the charge density constraint (2). Instead of the sublattice's order parameters it would be convenient to use their combinations, or analogues of the ferromagnetic and antiferromagnetic vectors:

$$
n=\frac{S_{A}+S_{B}}{2}, \quad S_{C O}=\frac{S_{A}-S_{B}}{2}, \quad \sigma_{F M}=\frac{\sigma_{A}+\sigma_{B}}{2}, \quad \sigma_{A F M}=\frac{\sigma_{A}-\sigma_{B}}{2} .
$$

Given $n=0$ there are (pseudo)antiferromagnetic solutions:

$$
S_{A}=-S_{B}=S, \quad \sigma_{A}=-\sigma_{B}=\sigma, \quad \xi=0,
$$

with a checkerboard charge $(\mathrm{CO})$ or spin $(\mathrm{AFM})$ ordering. In this case the mean field equations take the form as follows:

$$
\left\{\begin{aligned}
S & =\frac{\sinh 4 \nu S}{\cosh 4 \nu S+e^{\delta} \cosh j \sigma}, \\
\sigma & =\frac{\sinh j \sigma}{e^{-\delta} \cosh 4 \nu S+\cosh j \sigma} .
\end{aligned}\right.
$$

It should be emphasized that given nonzero doping $n \neq 0$ we arrive at a "(pseudo)ferrimagnetic" ground state $(\mathrm{CO}+\mathrm{FIM})$ with $S_{C O} \neq 0, \sigma_{F M} \neq 0$, and $\sigma_{A F M} \neq 0$ for a wide range of the values of the positive coupling parameters. 


\section{Phase diagrams and the temperature dependencies of the charge and spin order parameters}

The boundary between charge-ordered (CO) phase, where $S_{A} \neq S_{B}$, and non-ordered (NO) phase, where $S_{A}=S_{B}$, can be determined from the analysis of the mean field equations. Implicit equations of the CO-NO boundary for the $T-\Delta$ and $T-n$ phase diagrams are as follows

$$
1=4 \nu \frac{1+e^{\delta} \cosh \beta}{\left(e^{\delta}+\cosh \beta\right)^{2}}, \quad \beta=\log \frac{n e^{\delta}+\sqrt{1-n^{2}+n^{2} e^{2 \delta}}}{1-n} .
$$

These correspond to the second order phase transitions, if

$$
\sinh \beta\left(e^{2 \delta}-e^{\delta} \cosh \beta-2\right)(\beta+4 \nu n)<0,
$$

otherwise to the first order transitions.

Similarly, the boundary of antiferromagnetic (AFM) phase, where $\sigma_{A} \neq \sigma_{B}$, can be found. Its implicit equation for the $T-\Delta$ and $T-n$ phase diagrams reads as follows:

$$
1+e^{-\delta} \cosh \beta=j
$$

where $\beta$ is defined by Exp.(15). We arrive at the second order transition if $T>I / 3$, otherwise it is the first order transition.
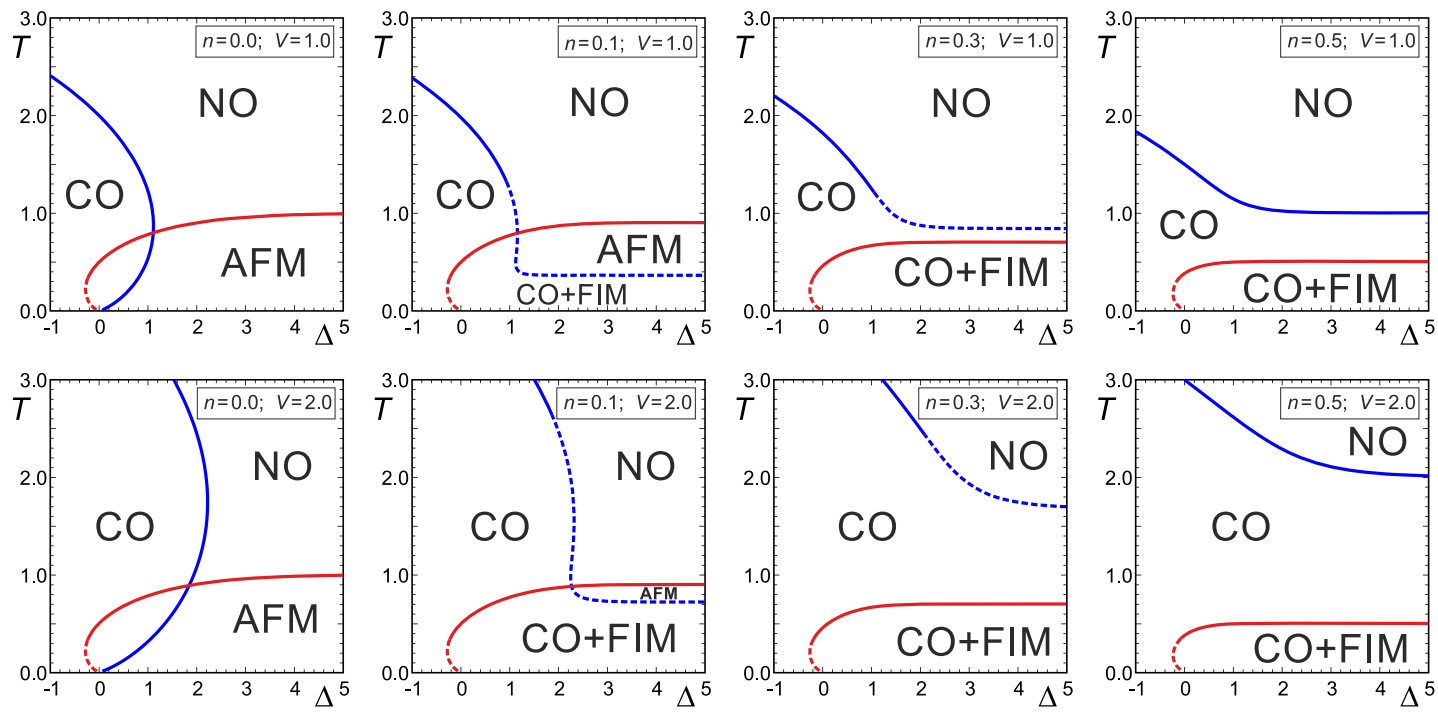

Figure 1: (Color online) $T-\Delta$ phase diagrams. The dashed lines correspond to first order transition, the solid ones correspond to second order transition (see text for detail).

The $T-\Delta$ and $T-n$ phase diagrams shown in Figs.1 and 2 were calculated using the numerical solution of the set of nonlinear coupled equations, Eqs.(15) and (17), given different doping and inter-site coupling parameters. It is worth to note, that strictly speaking the curves in Figs. 1 and 2 determine the boundaries of the phase stability. The dashed lines correspond to first order transition, the solid ones correspond to second order transition. The lines of 

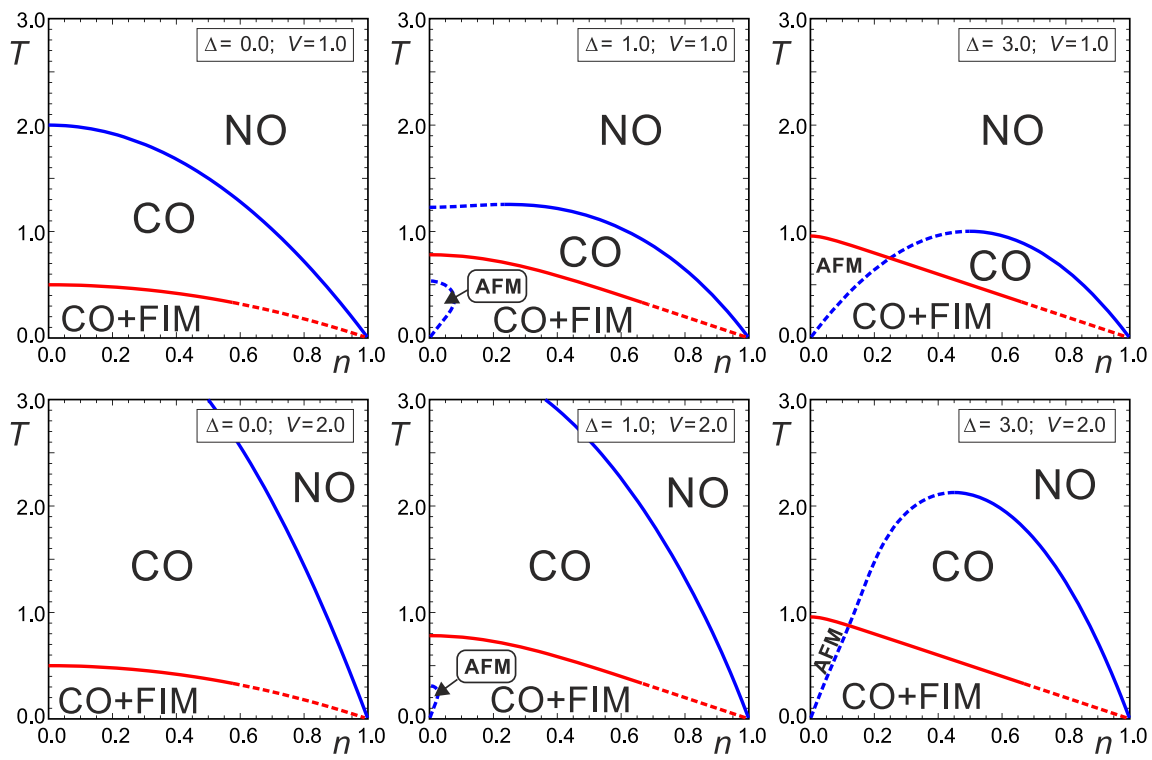

Figure 2: (Color online) $T-n$ phase diagrams. The dashed lines correspond to first order transition, the solid ones correspond to second order transition (see text for detail).

the first order phase transitions, that is the lines of equal potentials can be found with numerical minimization of grand potential. The temperature and coupling parameters here and below are given in units of the bare exchange integral $I$. Here NO denotes the non-ordered "(pseudo)paramagnetic" phase with an uniform charge distribution, $S_{A}=S_{B}=n$ but without the spin and charge order, $\sigma_{A}=\sigma_{B}=0\left(S_{C O}=0, \sigma_{A F M}=0, \sigma_{F M}=0\right)$.

At nonzero doping the checkerboard charge ordering in the CO phase where $S_{C O} \neq 0$ does coexist with a spin paramagnetism: $\sigma_{A}=\sigma_{B}=0$ while the checkerboard spin ordering in AFM phase where $\sigma_{A F M} \neq 0, \sigma_{F M}=0$ is accompanied by a "pseudo-spin paramagnetism": $S_{C O}=0$. For the low-temperature "(pseudo)ferrimagnetic" CO+FIM phases with coexistence of charge and spin orders we have $S_{A} \neq S_{B}, \sigma_{A} \neq \sigma_{B}$ or $\sigma_{A F M} \neq 0, \sigma_{F M} \neq 0, S_{C O} \neq 0$. The distinctive feature of the $\mathrm{CO}+\mathrm{FIM}$ phase consists in manifestation of the uncompensated spin magnetization.

As expected, at sufficiently high temperatures only fully nonordered NO phase survives. Interestingly that the transition into the fully ordered low-temperature $\mathrm{CO}+\mathrm{FIM}$ phase occurs only via intermediate "partially ordered" $\mathrm{CO}$ or AFM phases, the ordering phase transitions NO-CO and NO-AFM can be of the first or second order, depending on the magnitude of the coupling parameters and doping. The temperature of this transition can only weakly depend on the correlation parameters (at $\Delta>I$ ). As expected, the increase of the inter-site coupling $V>0$ does extend the region of the charge order, while the increase of the on-site repulsion does suppress the charge order stabilizing the low-temperature spin order.

We see a crucial effect of the doping under the charge density constraint (2). First, the rising doping does effectively suppress the parent AFM phase, so the phase survives only as a small island often being deep inside the CO+FIM phase. However, the charge ordering with $S_{A} \neq S_{B}$ survives even for large values of the correlation parameter $\Delta$ though this makes the active $S_{z}= \pm 1$ pseudospin states to be energy unfavorable ones. It should be noted that the doping dependence of the NO-CO transition temperature for a wide range of the on-site and 

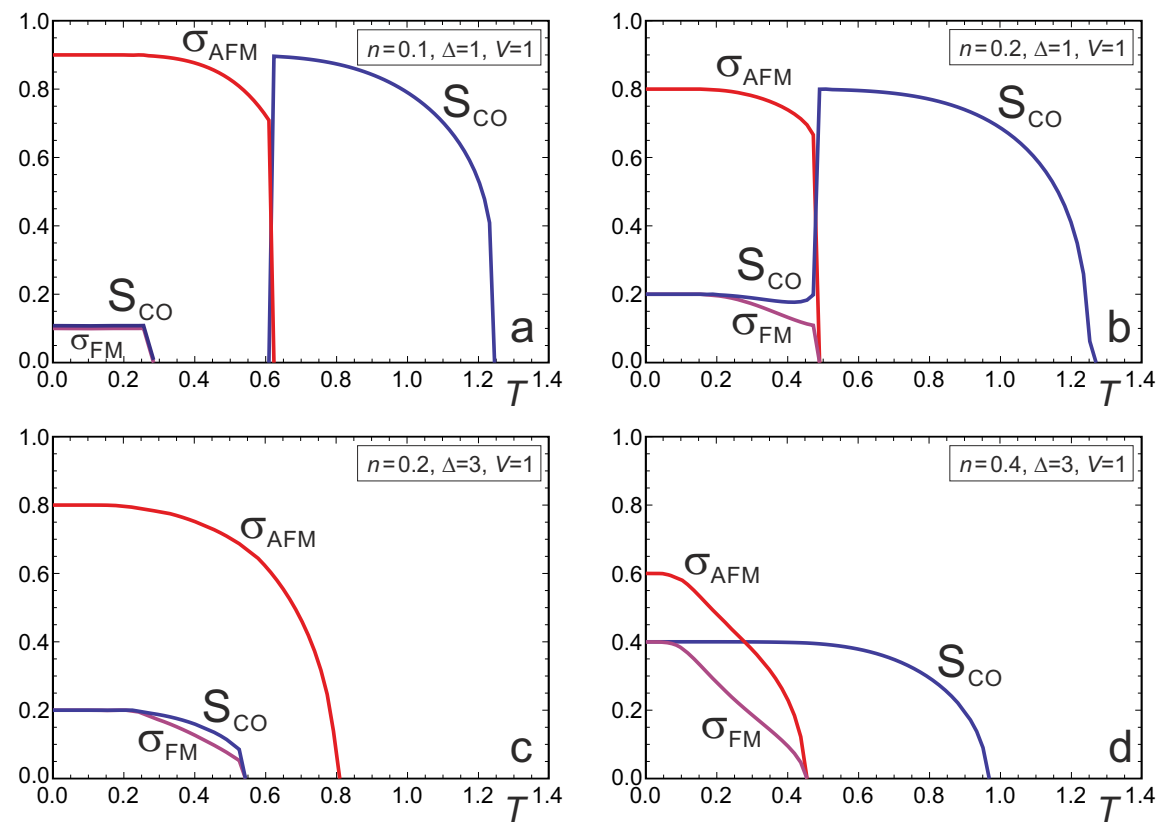

Figure 3: (Color online) Temperature dependence of the charge and spin order parameters at different values of doping $n$ and correlation parameter $\Delta$ (see text for detail).
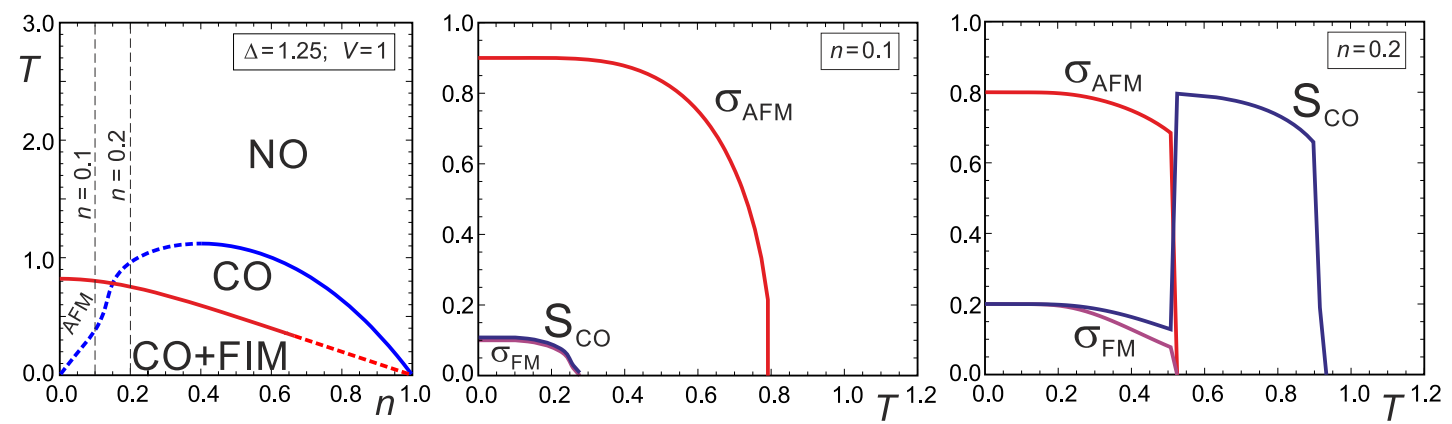

Figure 4: (Color online) $T-n$ phase diagram at $\Delta=1.25$ and the temperature behavior of the order parameters at $n=0.1$ and $n=0.2$. The dashed lines correspond to first order transition, the solid ones correspond to second order transition (see text for detail).

inter-site coupling parameters has much in common with that of a pseudogap temperature $T^{*}$ in real cuprates [1] that implies its relation with a some sort of charge ordering.

The Figs. 3 and 4 do illustrate the temperature behavior of the spin and charge order parameters calculated numerically on the assumption that the system follows the global minimum of the grand potential. We clearly see features typical for order parameters near phase transitions of the first or second order. Fig.3a shows temperature dependencies of the order parameters under a cascade of the sharp first-order phase transitions: $\mathrm{NO} \rightarrow \mathrm{CO} \rightarrow \mathrm{AFM} \rightarrow \mathrm{CO}+\mathrm{FIM}$. Figs.3b,c,d show similar dependencies for the phase transitions: $\mathrm{NO} \rightarrow \mathrm{CO} \rightarrow \mathrm{CO}+\mathrm{FIM}, \mathrm{NO} \rightarrow \mathrm{AFM} \rightarrow$ $\mathrm{CO}+$ FIM. The both Figs.3b,d concern with $\mathrm{NO} \rightarrow \mathrm{CO} \rightarrow \mathrm{CO}+$ FIM transition, however, with a 
clearly different character of the $\mathrm{CO} \rightarrow \mathrm{CO}+\mathrm{FIM}$ transition. It is worth noting that both spin and charge order parameters obey a "kinematic" constraint at $T=0:\left(\sigma_{F M}+\sigma_{A F M}\right)_{T=0}=1$ and $\left(n+S_{C O}\right)_{T=0}=1$ everywhere for AFM and CO phases, in fact, except the CO-FIM phase where the former relation continues to work while the latter breaks, instead, we arrive at $S_{C O}=\sigma_{F M}=n$ that evidences a crucial change of the spin-charge order with a full ferromagnetically ordered $B$-sublattice of $\mathrm{Cu}^{2+}$ ions $\left(S_{B}=0, \sigma_{B}=1\right)$ and magnetically diluted $A$-sublattice $\left(S_{A}=2 n, \sigma_{A}=2 n-1\right)$.

Given certain values of the model parameters we have observed effect of a dramatic change of spin-charge order under relatively minor change of the doping. This situation is shown in Fig.4. Given $n \leq 0.1$ the system displays mainly magnetic behavior, while at $n \approx 0.2$ the magnetic order becomes significantly suppressed with a sharp rise of the charge order. The situation resembles a well known suppression of the antiferromagnetic order with doping in cuprates [1].

\section{Conclusions}

We have analysed the competition between static charge and spin orders in a model cuprate using a two-sublattice mean-field approximation. Phase diagrams and temperature dependencies of the charge and magnetic order parameters are calculated for different doping given different values of the on-site correlation parameter, inter-site density-density coupling, and Heisenberg spin exchange integral. At variance with $t-J$-model [1] our model did emphasize the role of on-site and inter-site correlations and the occupation dependent spin exchange coupling in the competition of static spin and charge orders. Notwithstanding the fact that the present results have been obtained for a strongly simplified model, we have found that the evolution of the spin and charge orders with the model parameters and doping shares some common trends with real cuprates, so that the model can be used for study of the spin-charge competition and intertwinning effects. Obviously, that the taking account of one- and two-particle transport terms, long-range character of the inter-site Coulomb coupling, and inhomogeneous potential, typical for nonisovalently substituted cuprates, with going beyond MFA should be made for the genuine description of the competing orders in these materials. From the other hand, notwithstanding all the limitations, the model presented here is of interest in its own right, as an instructive tool to study an interplay of two competing orders.

Acknowledgements. The work was supported by the Government of the Russian Federation, Program 02.A03.21.0006 and by the Ministry of Education and Science of the Russian Federation, projects Nos. 1437 and 2725.

\section{References}

[1] Eduardo Fradkin, Steven A. Kivelson, and John M. Tranquada. Colloquium: Theory of intertwined orders in high temperature superconductors. Rev. Mod. Phys., 87:457-482, May 2015.

[2] A.S. Moskvin. True charge-transfer gap in parent insulating cuprates. Physical Review B, 84(7):075116, 2011.

[3] A.S. Moskvin. Pseudospin $s=1$ formalism and skyrmion-like excitations in the three-body constrained extended bose-hubbard model. JETP, 148(3):549, 2015.

[4] David C. Johnston. The puzzle of high temperature superconductivity in layered iron pnictides and chalcogenides. Advances in Physics, 59(6):803-1061, 2010. 